\title{
LETTER \\ Multilinear Supervised Neighborhood Embedding with Local Descriptor Tensor for Face Recognition
}

\author{
Xian-Hua HAN $^{\dagger \text { a) }}$, Xu QIAO ${ }^{\dagger}$, Nonmembers, and Yen-Wei CHEN ${ }^{\dagger}$, Member
}

\begin{abstract}
SUMMARY Subspace learning based face recognition methods have attracted considerable interest in recent years, including Principal Component Analysis (PCA), Independent Component Analysis (ICA), Linear Discriminant Analysis (LDA), and some extensions for 2D analysis. However, a disadvantage of all these approaches is that they perform subspace analysis directly on the reshaped vector or matrix of pixel-level intensity, which is usually unstable under illumination or pose variance. In this paper, we propose to represent a face image as a local descriptor tensor, which is a combination of the descriptor of local regions (K*K-pixel patch) in the image, and is more efficient than the popular Bag-Of-Feature (BOF) model for local descriptor combination. Furthermore, we propose to use a multilinear subspace learning algorithm (Supervised Neighborhood Embedding-SNE) for discriminant feature extraction from the local descriptor tensor of face images, which can preserve local sample structure in feature space. We validate our proposed algorithm on Benchmark database Yale and PIE, and experimental results show recognition rate with our method can be greatly improved compared conventional subspace analysis methods especially for small training sample number.

key words: tensor analysis, supervised neighborhood embedding, subspace learning, local SIFT feature, view-based object recognition
\end{abstract}

\section{Introduction}

Many face recognition techniques have been developed over the past few decades. One of the most successful and well-studied face recognition techniques is the appearancebased method [1], [2]. When using appearance-based methods, an image of size $n_{1} \times n_{2}$ pixels is usually represented by a vector in an $n_{1} \times n_{2}$-dimensional space. In practice, however, these $n_{1} \times n_{2}$-dimensional spaces are too large to allow robust and fast face recognition. Previous works have demonstrated that the face recognition performance can be improved significantly in lower dimensional linear subspaces [2]-[4]. Two of the most popular appearancebased face recognition methods include Eigenface [2] and Fisherface [3]. Eigenface is based on Principal Component Analysis (PCA) [5]. PCA projects the face images along the directions of maximal variances. It also aims to preserve the Euclidean distances between face images. Fisherface is based on Linear Discriminant Analysis (LDA) [5]. Unlike PCA which is unsupervised, LDA is supervised. When the class information is available, LDA can be used to find a linear subspace which is optimal for discrimination. Recently there are considerable interest in geometrically motivated approaches to visual analysis. Therein, the

Manuscript received May 28, 2010.

Manuscript revised September 6, 2010.

${ }^{\dagger}$ The authors are with College of Information Science and Engineering, Ritsumeikan University, Kusatsu-shi, 525-8577 Japan.

a)E-mail: hanxhua@fc.ritsumei.ac.jp

DOI: 10.1587/transinf.E94.D.158 most popular ones include Locality Preserving Projection (LPP) [4], Neighborhood Preserving Embedding (NPE) and so on, which can not only preserve the local structure between samples, and also obtain acceptable recognition rate for face recognition. In real application, all these subspace learning methods need to firstly reshape the 2D face image into 1D vector for analysis, which usually surfers "curse of dimension". Therefore, some researchers proposed to solve the "curse of dimension" problem with 2D subspace learning such as 2D-PCA, 2D-LDA [6] for analyzing directly on 2D image matrix, which was improved to be suitable in some extend. However, all of the conventional methods usually perform subspace analysis directly on the reshaped vector or matrix of pixel-level intensity, which would be unstable under illumination or pose variance.

In this paper, we propose to represent a face image as a local descriptor tensor, which is a combination of the descriptor of local regions ( $\mathrm{K}^{*} \mathrm{~K}$-pixel patch) in the image, and more efficient than the popular Bag-Of-Feature (BOF) model [7] for local descriptor combination. In order to extract discriminant feature from the local regions, we explore an improved gradient (intensity-normalized gradient) of the face image, which is robust to illumination variance, and use histogram of orientation weighed with the improved gradient for local region representation. Furthermore, we propose to use a multilinear subspace learning algorithm (or Tensor Supervised Neighborhood Embedding-TSNE) for discriminant feature extraction from the local descriptor tensor of face images, which can preserve local sample structure in feature space. Compared with tensorfaces [8] method which also directly analyze multi-dimensional data, the proposed TSNE uses supervised strategy, and thus can extract more discriminant features for distinguishing different objects (here facial images of different persons) and at the same time, can preserve samples' relationship of innerperson instead of only dimension reduction in tensorfaces. We validate our proposed algorithm on benchmark database Yale [3] and CMU PIE [9], and experimental results show recognition rate with our method can be greatly improved compared conventional subspace analysis methods especially for small training sample number.

The remaining parts of this paper are organized as follows. We introduce the local descriptor tensor for face images in Sect. 2. Section 4 propose a tensor supervised neighborhood embedding (TSNE) for extracting discriminant feature for face representation. Finally, we report experiment setup and results in Sect. 5, and give conclusion remarks in 
Sect. 6.

\section{Local Descriptor Tensor for Face Image Represen- tation}

In computer vision, local descriptors (i.e. features computed over limited spatial support) have proved well-adapted for matching and recognition tasks, as they are robust to partial visibility and clutter. The current popular one for local descriptor is SIFT feature, which is proposed by in [10] and is robust to small illumination variance. However with large illumination variance usually appeared in face recognition, it is still difficult to recognize correctly, and achieve acceptable recognition rate. Therefore, we proposed a histogram of orientation weighted with the improved gradient for local image representation. With the local descriptor, usually there are two types of algorithms for object recognition. One is to match the local point with SIFT feature in two images, and the other one is to use the popular Bag-Of-Feature model (BOF), which forms a frequency histogram of a predefined visual-words for all sampled region features [7]. For matching algorithm, it is usually not enough to recognize the unknown image even if there are several points well matched. How to combine more points feature is not unsolved still. The popular BOF model usually can achieve good recognition performance in most applications such as scene and object recognition. However, in BOF model, in order to achieve acceptable recognition rate it is necessary to sample a lot of points for extracting SIFT features (usually more than 1000 in an image), and compare the extracted local feature with the predefined visual-words (usually more than 1000) to obtain the visual-word occurrence histogram. Therefore, BOF model need a lot of computing time to extract visual-words occurrence histogram. In addition, BOF model just approximately represent each local region feature as the predefined visual-words, and then, it maybe lose a lot of information and will be not efficient for image representation. Therefore, in this paper, we propose to represent a face image as a combined local descriptor tensor.

In order to extract robust feature to illumination variance, we need to obtain the improved gradient. Given an image I, we calculate the improved gradient (Intensitynormalized gradient) using the following Eq.:

$$
\begin{aligned}
& \mathbf{I}_{x}(i, j)=\frac{\mathbf{I}(i+1, j)-\mathbf{I}(i-1, j)}{\mathbf{I}(i+1, j)+\mathbf{I}(i-1, j)} \\
& \mathbf{I}_{y}(i, j)=\frac{\mathbf{I}(i, j+1)-\mathbf{I}(i, j-1)}{\mathbf{I}(i, j+1)+\mathbf{I}(i, j-1)} \\
& \mathbf{I}_{x y}(i, j)=\sqrt{\mathbf{I}_{x}(i, j)^{2}+\mathbf{I}_{y}(i, j)^{2}}
\end{aligned}
$$

where $\mathbf{I}_{x}(i, j)$ and $\mathbf{I}_{y}(i, j)$ means the horizontal and vertical gradient in pixel position $i, j$, respectively, $\mathbf{I}_{x y}(i, j)$ means the global gradient in pixel position $i, j$. The idea of the normalized gradient is from $\chi^{2}$ distance: a normalized $\mathrm{Eu}-$ clidean distance. For $x$-direction, the gradient is normalized by summation of the upper one and the bottom one pixel

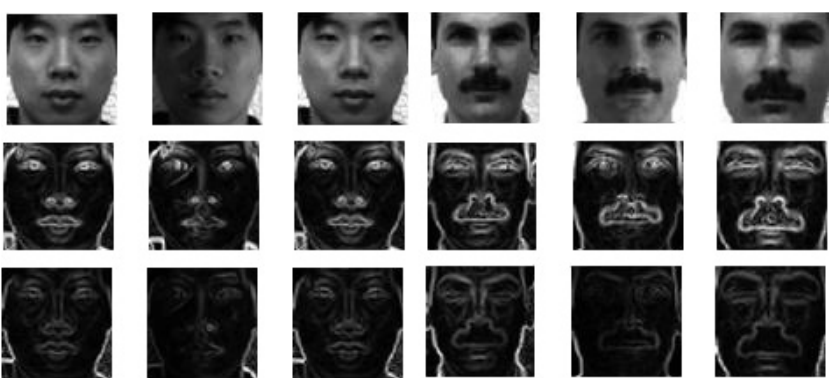

Fig. 1 Gradient image samples. Top row: Original face images; Middle row: the intensity-normalized gradient images; Bottom row: the conventional gradient images.

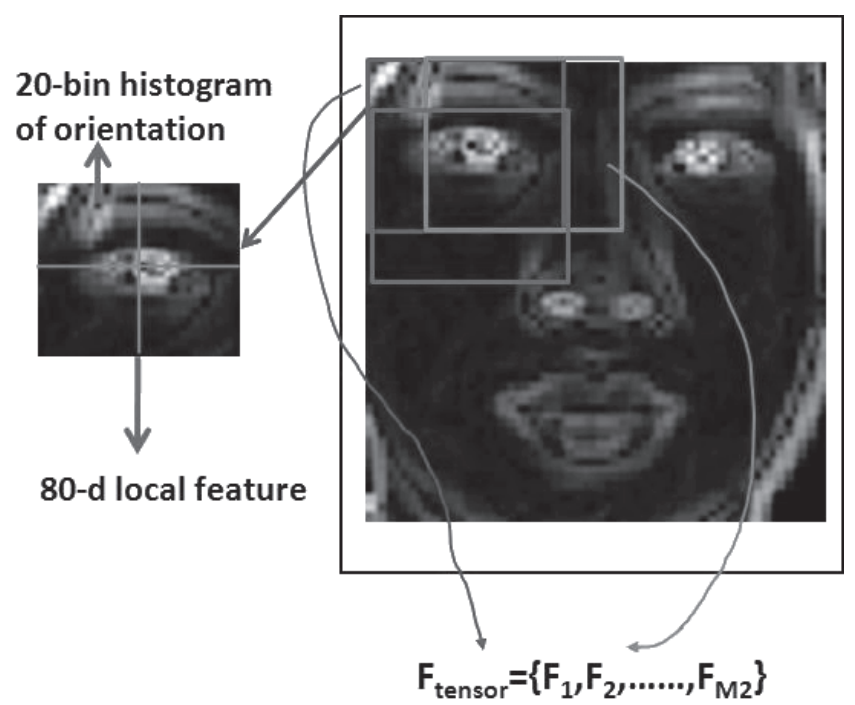

Fig. 2 Extraction procedure of local descriptor tensor from a face image. The top-left rectangle in the right part of this figure is the first extracted region for calculating local descriptor (a 80-bin edge histogram); The topright rectangle is the next extracted region after moving several pixels from the top-left one (predefined interval) along row, and continue this step until the end of row pixels. The bottom rectangle is the first extracted region after moving several pixels for the top-left one along column, and then obtain next regions through moving pixel in row. The total number of extracted regions is $M 2$.

centered by the focused pixel; for $y$-direction, the gradient is normalized by that of the right and left one. With the intensity-normalized gradient, we can extract robust and invariant features to illumination changing in a local region of an image. Some examples with the intensity-normalized and conventional gradients are shown in Fig. 1

(1) The feature extraction of local regions (local descriptor): given a local region $I^{R}$ in an face image, we firstly segment the region into $4(2 \times 2)$ patches, and in each patch, we extract a 20-bin histogram of orientation weighted by global gradient using the intensity-normalized gradients $\mathbf{I}_{x}^{R}$, $\mathbf{I}_{y}^{R}$ and $\mathbf{I}_{x y}^{R}$. Therefore, each region in a face image can be represent by 80 -bin $(20 \times 4)$ histogram as shown in the left part of Fig. 2.

(2) In order to efficiently represent a face image, we grid-segment an image, and can obtain $M 2$ overlapping regions as shown in the right part of Fig. 2, and then in each 
region, we extract a 80-bin histogram feature (1D tensor). Furthermore we combine the $M 2$ vectors (local descriptors) into a $2 \mathrm{D}$ tensor with of size $80 \times M 2$ in the space $R_{80} \otimes R_{M 2}$ for representing a face image. The tensor feature extraction procedure of a face image is shown in Fig. 2.

\section{Tensor Supervised Neighborhood Embedding}

In order to model N-D data without rasterization (2D is a special case), tensor representation is proposed and analyzed for feature extraction or modeling. In this section, we propose a tensor supervised neighborhood embedding to not only extract discriminant feature but also preserve the local geometrical and topological properties in same category for recognition. The proposed approach decompose each model of tensor with objective function, which consider neighborhood relation and class label of training samples.

Suppose we have ND tensor objects $\mathcal{X}$ from $C$ classes. The $c^{\text {th }}$ class has $n^{c}$ tensor objects and the total number of tensor objects is $n$. Let $X_{i_{c}} \in R^{N_{1}} \otimes R^{N_{2}} \otimes \cdots \otimes R^{N_{L}}\left(i_{c}=\right.$ $\left.1,2, \cdots, n^{c}\right)$ be the $i^{\text {th }}$ object in the $c^{\text {th }}$ class. For a gray face image, we can directly represent it as pixel-level intensity tensor, where $L$ is $2, N_{1}$ is the row number, $N_{2}$ is the column number. We also can represent the face image as a feature-based tensor such as local descriptor feature tensor introduced in Sect. 2, where $L$ is also $2, N_{1}$ is the local feature dimension, $N_{2}$ is the sampled region number in an image. Then, we can build a nearest neighbor graph $\mathcal{G}$ to model the local geometrical structure and label information of $\mathcal{X}$. Let $W$ be the weight matrix of $\mathcal{G}$. A possible definition of $W$ is as follows:

$$
W_{i j}= \begin{cases}\exp ^{-\frac{x_{i}-X_{j}}{t}} & \text { if sample } i \text { and } j \text { are in same class } \\ 0 & \text { otherwise }\end{cases}
$$

Let $\mathbf{U}_{d}$ be the $d$-model transformation matrices (Dimension: $\left.N_{d} \times D_{d}\right)$. A reasonable transformation respecting the graph structure can be obtained by solving the following objective functions:

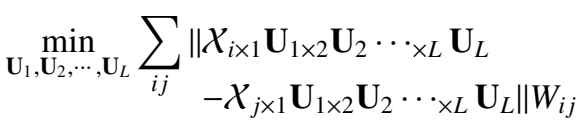

The objective function incurs a heavy penalty if neighboring points $\mathcal{X}_{i}$ and $\mathcal{X}_{j}$ are mapped far apart. Therefore, minimizing it is an attempt to ensure that if $\mathcal{X}_{i}$ and $\mathcal{X}_{j}$ are "close", then $\mathcal{X}_{i \times 1} \mathbf{U}_{1 \times 2} \mathbf{U}_{2} \cdots_{\times L} \mathbf{U}_{L}$ and $\mathcal{X}_{j \times 1} \mathbf{U}_{1 \times 2} \mathbf{U}_{2} \cdots_{\times L} \mathbf{U}_{L}$ are "close" as well. Let $\boldsymbol{y}_{i}=$ $\mathcal{X}_{i \times 1} \mathbf{U}_{1 \times 2} \mathbf{U}_{2} \cdots \times L \mathbf{U}_{L}$ (Dimension: $D_{1} \times D_{2} \times \cdots \times D_{L}$ ), and $\left(\mathbf{Y}_{i}\right)^{d}=\left(\mathbf{X}_{i \times 1} \mathbf{U}_{1 \times 2} \mathbf{U}_{2} \cdots_{\times L} \mathbf{U}_{L}\right)^{d}$ (2D matrix, Dimension: $\left.D_{d} \times\left(D_{1} \times D_{2} \times \cdots \times D_{d-1} \times D_{d+1} \times \cdots \times D_{L}\right)\right)$ is the dmode extension of tensor $\boldsymbol{Y}_{i}$. Let $\mathrm{D}$ be a diagonal matrix, $D_{i i}=\sum_{j} W_{i j}$. Since $\|\mathbf{A}\|^{2}=\operatorname{tr}\left(\mathbf{A} \mathbf{A}^{T}\right)$, we see that

$$
\frac{1}{2} \sum_{i j}\left\|X_{i \times 1} \mathbf{U}_{1} \cdots_{\times L} \mathbf{U}_{L}-\mathcal{X}_{j \times 1} \mathbf{U}_{1} \cdots_{\times L} \mathbf{U}_{L}\right\| W_{i j}
$$

$$
\begin{aligned}
& =\frac{1}{2} \sum_{i j} \operatorname{tr}\left(\left(\left(\mathbf{Y}_{i}\right)^{d}-\left(\mathbf{Y}_{j}\right)^{d}\right)\left(\left(\mathbf{Y}_{i}\right)^{d}-\left(\mathbf{Y}_{j}\right)^{d}\right)^{T}\right) W_{i j} \\
& =\operatorname{tr}\left(\sum_{i} D_{i i}\left(\mathbf{Y}_{i}\right)^{d}\left(\left(\mathbf{Y}_{i}\right)^{d}\right)^{T}-\sum_{i j} W_{i j}\left(\mathbf{Y}_{i}\right)^{d}\left(\left(\mathbf{Y}_{j}\right)^{d}\right)^{T}\right) \\
& =\operatorname{tr}\left(\sum _ { i } D _ { i i } \left(\mathbf{U}_{d}^{T}\left(X_{i \times 1} \mathbf{U}_{1} \cdots_{\times d-1} \mathbf{U}_{d-1 \times d+1} \mathbf{U}_{d+1} \cdots_{\times L} \mathbf{U}_{L}\right)\right.\right. \\
& \left(X_{i \times 1} \mathbf{U}_{1} \cdots_{\times d-1} \mathbf{U}_{d-1 \times d+1} \mathbf{U}_{d+1} \cdots_{\times L} \mathbf{U}_{L}\right)^{T} U_{d} \\
& -\sum_{i j} W_{i j}\left(\mathbf{U}_{d}^{T}\left(X_{i \times 1} \mathbf{U}_{1} \cdots_{\times d-1} \mathbf{U}_{d-1 \times d+1} \mathbf{U}_{d+1} \cdots_{\times L} \mathbf{U}_{L}\right)\right. \\
& \left.\left.\quad\left(X_{j \times 1} \mathbf{U}_{1} \cdots_{\times d-1} \mathbf{U}_{d-1 \times d+1} \mathbf{U}_{d+1} \cdots_{\times L} \mathbf{U}_{L}\right)^{T} U_{d}\right)\right) \\
& \operatorname{tr}\left(\mathbf { U } _ { d } ^ { T } \left(\sum_{i} D_{i i}\left(X_{i \times 1} \mathbf{U}_{1} \cdots_{\times d-1} \mathbf{U}_{d-1 \times d+1} \mathbf{U}_{d+1} \cdots_{\times L} \mathbf{U}_{L}\right)\right.\right. \\
& \quad\left(X_{i \times 1} \mathbf{U}_{1} \cdots_{\times d-1} \mathbf{U}_{d-1 \times d+1} \mathbf{U}_{d+1} \cdots_{\times L} \mathbf{U}_{L}\right)^{T} \\
& -\sum_{i j} W_{i j}\left(X_{i \times 1} \mathbf{U}_{1} \cdots_{\times d-1} \mathbf{U}_{d-1 \times d+1} \mathbf{U}_{d+1} \cdots_{\times L} \mathbf{U}_{L}\right) \\
& \left.\left.\quad\left(X_{j \times 1} \mathbf{U}_{1} \cdots_{\times d-1} \mathbf{U}_{d-1 \times d+1} \mathbf{U}_{d+1} \cdots_{\times L} \mathbf{U}_{L}\right)^{T}\right) U_{d}\right) \\
& =\operatorname{tr}\left(\mathbf{U}_{d}^{T}\left(\mathbf{D}_{d}-\mathbf{S}_{d}\right) U_{d}\right)
\end{aligned}
$$

where $\mathbf{D}_{d}=\sum_{i} D_{i i}\left(\left(X_{i \times 1} \mathbf{U}_{1} \cdots_{\times d-1} \mathbf{U}_{d-1 \times d+1} \mathbf{U}_{d+1} \cdots_{\times L} \mathbf{U}_{L}\right)\right.$ $\left.\left(X_{i \times 1} \mathbf{U}_{1} \cdots_{\times d-1} \mathbf{U}_{d-1 \times d+1} \mathbf{U}_{d+1} \cdots_{\times L} \mathbf{U}_{L}\right)^{T}\right)$ and $\mathbf{S}_{d}=\sum_{i j} W_{i j}$ $\left(\left(X_{i \times 1} \mathbf{U}_{1} \cdots_{\times d-1} \mathbf{U}_{d-1 \times d+1} \mathbf{U}_{d+1} \cdots_{\times L} \mathbf{U}_{L}\right)\left(X_{j \times 1} \mathbf{U}_{1} \cdots_{\times d-1}\right.\right.$ $\left.\left.\mathbf{U}_{d-1 \times d+1} \mathbf{U}_{d+1} \cdots_{\times L} \mathbf{U}_{L}\right)^{T}\right)$. Therefore the linear transformation $\mathbf{U}_{d}$ can be obtained by minimizing the objective function under constraint:

$$
\mathbf{U}_{d}=\underset{\mathbf{U}_{d}^{T} \mathbf{D}_{d} \mathbf{U}_{d}=1}{\arg \min }\left(\mathbf{U}_{d}^{T}\left(\mathbf{D}_{d}-\mathbf{S}_{d}\right) U_{d}\right)
$$

In order to achieve the stable solution, we firstly regularize the symmetric matrix $\mathbf{D}$ as $D_{i i}=D_{i i}+\alpha$ ( $\alpha$ is a small value). Finally, the minimization problem can be converted to solving a generalized eigenvalue problem as follows:

$$
\mathbf{D}_{d} U_{d}=\lambda \mathbf{S}_{d} U_{d}
$$

After obtaining the TSNE basis of each mode, we can project each tensor object into these TSNE basis for each mode. For face recognition, the projection coefficients can represent the extracted feature vectors and can be used for classification using Euclidean distance or other similar measurement.

\section{Experimental Results}

In this paper, we use the benchmark face dataset YALE, which includes 15 people and 11 facial images of each individual with different illuminations and expressions, and CMU PIE, which includes 68 people and about 170 facial images for each individual with 13 different poses, 43 different illumination conditions, and with 4 different expressions. For YALE dataset, we randomly select 2, 3, 4 
Table 1 Average recognition error rates (\%) on YALE dataset with different training number.

\begin{tabular}{lrrrr}
\hline Method & 2 Train & 3 Train & 4 Train & 5 Train \\
\hline PCA & 56.5 & 51.1 & 57.8 & 45.6 \\
\hline LDA & 54.3 & 35.5 & 27.3 & 22.5 \\
\hline Laplacianface & 43.5 & 31.5 & 25.4 & 21.7 \\
\hline O-Laplacianface & 44.3 & 29.9 & 22.7 & 17.9 \\
\hline TensorLPP & 54.5 & 42.8 & 37 & 32.7 \\
\hline R-LDA & 42.1 & 28.6 & 21.6 & 17.4 \\
\hline S-LDA & 37.5 & 25.6 & 19.7 & 14.9 \\
\hline TSNE & 41.89 & 31.67 & 24.86 & 23.06 \\
\hline TSNE-SIFT & 35.22 & 26.33 & 22.19 & 20.83 \\
\hline TSNE-NHOG & $\mathbf{2 9 . 7 4}$ & $\mathbf{2 2 . 8 7}$ & $\mathbf{1 8 . 5 2}$ & $\mathbf{1 7 . 4 4}$ \\
\hline
\end{tabular}

Table 2 Average recognition error rates (\%) on YALE dataset with different training number.

\begin{tabular}{lrr}
\hline Method & 5 Train & 10 Train \\
\hline PCA & 75.33 & 65.5 \\
\hline LDA & 42.8 & 29.7 \\
\hline LPP & 38 & 29.6 \\
\hline TSNE & 37.66 & 23.57 \\
\hline TSNE-NHOG & $\mathbf{3 3 . 8 5}$ & $\mathbf{2 2 . 0 6}$ \\
\hline
\end{tabular}

and 5 facial images from each individual for training, and the remainders for test. We do 20 runs for different training number and average recognition rate. For comparison, we also do experiments using the proposed TSNE analysis on directly the gray face image (pixel-level intensity, denoted TSNE), local feature tensor with SIFT descriptor (denoted TSNE-SIFT) and our proposed intensity-normalized histogram of orientation (denoted TSNE-NHOG). The compared results are shown in Table 1 using TSNE analysis with different tensors and other subspace learning methods by $\mathrm{He}$ [4], [11], [12]. From Table 1, it is obvious that our proposed algorithm can obtain the best recognition performance especially using small training samples. For CMU PIE dataset, we randomly select 5 and 10 facial images from each individual for training, and the remainder for test. We also do 20 runs for achieving average recognition error rate. The compared recognition error rates between our proposed algorithms and the conventional subspace learning methods by He [4], [11], [12] are shown in Table 2.

\section{Conclusions}

In this paper, we proposed to represent a face image as a local descriptor tensor, which is a combination of the descriptor of local regions ( $\mathrm{K}^{*} \mathrm{~K}$-pixel patch) in the image, and more efficient than the popular Bag-Of-Feature (BOF) model for local descriptor combination. Furthermore, we proposed to use Supervised Neighborhood Embedding (SNE) for discriminant feature extraction from the local descriptor tensor of face images, which can preserve local sample structure in feature space. We validate our proposed algorithm on Benchmark database Yale and PIE, and experimental results show recognition rate with our method can be greatly improved compared conventional subspace analysis methods especially for small training sample numbers.

\section{References}

[1] H. Murase and S.K. Nayar, "Visual learning and recognition of 3-d objects from appearance," Int. J. Comput. Vis., vol.14, no.1, pp.524, 1995.

[2] M. Turk and A. Pentland, "Eigenfaces for recognition," J. Cognitive Neuroscience, vol.3, no.1, pp.71-86, 1991.

[3] P.N. Belhumeur, J.P. Hepanha, and D.J. Kriegman, "Eigenfaces vs. fisherfaces: Recognition using class specific linear projection," IEEE Trans. Pattern Anal. Mach. Intell., vol.19, no.7, pp.711-720, 1997.

[4] X. He, S. Yan, Y. Hu, P. Niyogi, and H.-J. Zhang, "Face recognition using Laplacianfaces," IEEE Trans. Pattern Anal. Mach. Intell., vol.27, no.3, pp.328-340, 2005.

[5] R.O. Duda, P.E. Hart, and D.G. Stork, Pattern Classification, 2nd ed., Wiley-Interscience, Hoboken, NJ, 2000.

[6] X.-M. Wang, C. Huang, X.-Y. Fang, and J.-G. Liu, "2DPCA vs. 2DLDA: Face recognition using two-dimensional method," International Conference on Artificial Intelligence and Computational Intelligence, vol.2, pp.357-360, 2009.

[7] G. Csurka, C. Dance, L. Fan, J. Willamowski, and C. Bray, "Visual categorization with bags of keypoints," Proc. ECCV Workshop on Statistical Learning in Computer Vision, pp.1-16, 2004.

[8] M.A.O. Casilescu and D. Terzopoulos, "Multilinear analysis of image ensembles: TensorFaces," ECCV, 2002.

[9] T. Sim, S. Baker, and M. Bsat, "The CMU Pose, Illumination, and Expression (PIE) database of human faces," Robotics Institute, CMU-RI-TR-01-02, Pittsburgh, PA, 2001.

[10] D. Lowe, "Distinctive image features from scale-invariant keypoints," Int. J. Comput. Vis., vol.60, no.2, pp.91-110, 2004.

[11] D. Cai, X. He, Y. Hu, J. Han, and T. Huang, "Learning a spatially smooth subspace for face recognition," CVPR'07, 2007.

[12] D. Cai, X. He, and J. Han, "Spectral regression for efficient regularized subspace learning," ICCV’07, 2007. 